POLSKA AKADEMIA NAUK-ZAKEAD BADANIA SSAKOW

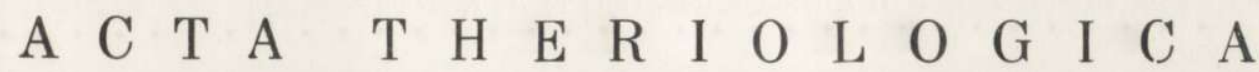
VOL. IX, 4.

\title{
The Rate of Passage of Foodstuifs through the Alimentary Tracts of Certain Microtidae Under Laboratory Conditions
}

\author{
Szybkość przechodzenia treści przez przewód pokarmowy \\ niektórych Microtidae w warunkach laboratoryjnych
}

[With 5 Tables \& 3 Figs.]

I. Introduction

II. Investigation methods

III. Results

1. Passage of the green parts of plants . . . . . . . . . . 40

2. Passage of seeds. . . . . . . . . . . . . . . 42

3. Comparison of the rate of passage of the green parts of plants with the rate of passage of seeds

4. Sex dimorphism in the rate of passage of foodstuffs . . . . . . 48

IV. Discussion . . . . . 49

V. Summary . . . . . . . . . 50

References . . . . . . . . . . . . . . . . 50

Streszczenie . . . . . . . . 53

\section{INTRODUCTION}

The rate of passage of foodstuffs through the alimentary tract has long since aroused the interest of research workers, primarily on account of the importance of this problem in the rearing of animals and also in research on metabolism. A very considerable amount of data is available in literature on the rate of passage of dif-ferent foodstuffs through the alimentary tract of domestic animals and those intended for industrial purposes (Lenkeit \& Habeck, 1930; Lenkeit, 1931; Ea l ch, 1950; Mangold \& Behm, 1954; Neseni, Lecht \& S chewen, 1955; Cas t le, 1956; Castle \& Castle, 1956, 1957; Neseni \& Piatkowski, 1958; Koźniewski, 1961; Horszczaruk, 1962; S ławiński, Bednarz \& Sławoń, 1962; S ła wińs ki, S ła woń \& Bedn a r z, 1962). A few authors have investigat-ed this problem in relation to wild animals ( $\mathrm{H}$ on ig m a n, 1956; G i 11, 1957, 1959, 
1960), in particular rodents (Velitshko \& Mokejeva, 1949; Velitshko, 1956; Pravdina, 1958; Gill \& Bieguszewski, 1960). A better knowledge of the course taken by this process in Rodentia is interesting on account of the specific structure of the alimentary tract in these mammals and the relative ease with which the rapid evolution connected with the transition from the feeding on protein food to cellulose food can be traced in these animals (V or o n ts ov, 1962).

Our work was therefore aimed at investigating and comparing the rate of passage of foodstuffs through the alimentary tract of certain Microtidae differing as to the kind of food they eat (seeds, green plants) and as to the structure of the alimentary system. It was also decided to trace the course taken by this process in both sexes of these rodents. The investigations were made on Clethrionomys glareolus $(\mathrm{S} \mathrm{c} \mathrm{h} \mathrm{r} \mathrm{e-}$ be r, 1780), Microtus agrestis L in n a e s, 1761, Microtus arvalis ( $\mathrm{P}$ a 11 a s, 1779) and Microtus nivalis $\mathrm{M}$ artins, 1842, which belong to two wel! distinguished genera of Microtidae (Table 1).

$\mathrm{H}$ a r de $\mathrm{r}$ (1950) divided rodents into those feeding on seed food and those on the green parts of plants, while Vor on ov (1954) distinguished yet another group of Rodentia, the omnivorous group. $C$. glareolus represents the group of which the basic food is formed by seeds (N a u m ov, 1948; M ille r, 1954; V or on ts ov, 1961; Górecki \& Gębczyńska, 1962), and M. arvalis is a typical representative of the rodents which feed on the green parts of plants ( $\mathrm{Na} \mathrm{u} \mathrm{mov}, 1948$; G ole $\mathrm{n}$ is tschev, 1952; Kagantsova, 1954; Voronov, 1954; B a shenina, 1962). M. agrestis feeds on both grain and the green parts of plants, with a slight preference for grain according to $\mathrm{N}$ a u mov (1948), and according to $\mathrm{N}$ a S a r o v a (1958). a preference for green plants. We know from literature that $M$. nivalis feeds chiefly on the green parts of plants (B a uman, 1949; O g nev, 1950; Z i m m e r ma n n, 1959; K ow a ls k i, 1964), but there are no detailed data on the composition of the food of this species.

The different food relations of the species of rodents referred to are reflected in the structure of their digestive systems. Numerous authors maintain that Rodentiu, the basic food of which is formed by green plants, have a very strongly developed caecum and large intestine ( $\mathrm{Na} \mathrm{u} \mathrm{mov}, 1948$; $\mathrm{H}$ a r de r, 1950; S chwarz, 1960; Vor ontsov, 1962), in which the digestion of cellulose, with the participation of specific microflora, takes place (Velitshko \& Mokejeva, 1949; Golley, 1960). Rodents feeding on seed foods, on the other hand, have a shorter posterior part of the intestine, and longer small intestine, in which starch and protein are digested ( $\mathrm{Na} \mathrm{umov}, 1948 ; \mathrm{Kula}$ jeva, 1958; Voron ts ov, 1962). Of the animals examined $M$. arvalis has the most strongly developed caecum and large intestine, and a relatively short small intestine; in the case of $M$. agrestis the small intestine is slightly longer and the posterior part of the digestive system shorter ( $\mathrm{N}$ a s a r o$\mathrm{v}$ a, 1958); in C. glareolus the relative lengths of each section of the intestines form. the opposite of the above - the small intestine is the longest, and the caecum an 1 large intestine shortest ( $\mathrm{N}$ a u m ov, 1948; $\mathrm{Kulajeva,1958).}$

\section{INVESTIGATION METHODS}

Use was made in our experiments of the stained food method worked out by Lenkeit \& Habeck (1930) and Mangold (1950), improved by Castle (1956) and modified by Gill (1957), using basic fuchsin as the colouring agent. In the 
experiments concerned with the passage of the green parts of plants, $M$. arvalis, $M$. agrestis and $M$. nivalis were fed with Arrhenatherum elatius L., Trifolium sp. and Taraxacum officinale $\mathrm{We}$., and $\mathrm{C}$. glareolus received Viola silvestris $\mathrm{R} \mathrm{chb}$., Oxalis acetosella L., Pulmonaria obscura D um. - defined by Górecki \& Gę b. c z y ńs k a (1962) as some of the species of plants most readily eaten by this rodent, and also $1 \mathrm{~g}$. of wheat grain. The addition of grain proved essential, as $C$. glareolus was found to lose weight and die when kept on an exclusively green diet. This was the cause of the limited number of animals in this experiment. During the experiments on the rate of passage of grain through the alimentary tract in all four species of Microtidae examined, the animals were fed with wheat grain. Since under natural conditions acorns form one of the basic components of the food of C. glareolus (G órecki \& Gębczynska, 1962), their rate of passage through the alimentary system of these animals was also examined for purposes of comparison with the rate of passage of wheat. The indicator of the passage of foodstuffs through the alimentary tract was always the stained portion of the food with which the animals were fed during the experiment. They were given the stained food at 10 a.m., and this was followed after one hour by a similar, but not stained, portion of food.

Table 1.

Number of individuals examined.

\begin{tabular}{|c|c|c|c|c|c|c|c|c|}
\hline Spectes & \multicolumn{2}{|c|}{ M. arval1s } & \multicolumn{2}{|c|}{ N. agrestis } & \multicolumn{2}{|c|}{ C. glareolus } & \multicolumn{2}{|c|}{ u. n1val1s } \\
\hline K1nd of food & 86 & $\$ q$ & 86 & $\$ 9$ & of & q9 & 68 & q? \\
\hline Green parts of plants & 15 & 15 & 15 & 15 & 6 & 3 & 5 & 2 \\
\hline Wheat gra1ns & 15 & 15 & 15 & 15 & 15 & 15 & 4 & 2 \\
\hline Acorns & - & - & - & - & 4 & 3 & - & - \\
\hline Total & \multicolumn{2}{|c|}{60} & \multicolumn{2}{|c|}{60} & \multicolumn{2}{|c|}{46} & \multicolumn{2}{|c|}{13} \\
\hline
\end{tabular}

Excrement was collected at intervals of one hour, then dried at a temperature of about $60^{\circ} \mathrm{C}$, and each portion weighed on a torsion balance with accuracy of up to $0.5 \mathrm{mg}$., then diluted with $0.02 \mathrm{ml}$. of water to each mg., and thoroughly mixed. The stained food was calculated in each sample in five fields of vision of the microscope, magnified 150 times, using the Fuchs-Rosenthal camera ensuring that layers of uniform thickness were obtained. A total of 8000 samples of excrement were examined. The total amount of stained food calculated in all the samples coming from one animal were taken as $100 \%$, then calculation was made of the percentage of test food excreted in each hour of the experiment. Taking these data as a basis, curves were drawn according to $\mathrm{Bal} \mathrm{ch}$ (1950) illustrating the course taken by the passage of food through the alimentary tract of the animals examined in each of the experiments. $R$ values (C a stle, 1956), being the mean time of retention of food in the digestive system of the animals and enabling a comparisori of the curves throughout their entire length to be made, were determined, and calculation made of the times of excretion of $5 \%, 50 \%, 90 \%$ and $100 \%$ of the stained food in each of the experiments. All these values were compared by the $t$-Student test for the difference in the mean values for two independent groups, and calculation made for them of the divisions of confidence based on the variable $t$.

The animals were placed singly in wooden cages measuring $40 \times 15.5 \times 17.5 \mathrm{~cm}$., from 4 to 7 days before the beginning of the experiment. Each cage contained 
a wooden six-sided house, each side measuring $9 \mathrm{~cm}$. in width, filled with paper shavings. These houses served as nests during the preparatory period. During the actual experiment the houses were taken out of the cages. A metal net was placed at a height of $1.5 \mathrm{~cm}$. above the bottom of each cage, which permitted of the frequent collection of excrement without disturbing the rodents. The animals were fed on the same kind of food both during the preparatory period and during the experiment.

During the experiments endeavour was made to maintain uniform temperature and humidity conditions in the places in which the rodents under examination were kept (temperature about $20^{\circ} \mathrm{C}$, humidity about $80 \%$ ).

\section{RESULTS}

\section{Passage of the green parts of plants (Tables 2, 4; Fig. 1)}

The process of excretion of the indicator began in all the species examined in the first hour of the experiment, and was completely finished after the lapse of $17-18$ hours. C. glareolus excreted $50 \%$ of the indicator most rapidly $(34.80 \% /$ hour), $M$. agrestis more slowly $(25.20 \% /$ hour $)$ and $M$. arvalis $(18.60 \% /$ hour) and $M$. nivalis $(14.40 \% /$ hour) the slowest. With this distribution of passage rate the main mass of the test food also passes through the alimentary tract of $C$. glareolus most rapidly, that is, after $3-3.5$ hours. In the case of $M$. agrestis this process takes slightly longer (3.5-4 hours), while it is slowest in the case of $M$. arvalis (4-4.5 hours) and $M$. nivalis (5-6 hours). After the passage of the main mass of the indicator the rate of excretion gradually decreased in all the species and the lowest value was attained with the excretion of: the final $10 \%$ of stained food (about 1\%/hour). The differences discussed between the species of animal examined, occurring in the rate of excretion of the test food, are reflected in the $R$ values, which are lower in $M$. agrestis and C. glareolus in comparison with $M$. arvalis and $M$. nivalis (Table 2).

Comparison of $R$ values and excretion times of $5 \%, 50 \%, 90 \%$ and $100 \%$ of the indicator revealed statistically significant differences in many cases (Table 3). The statistical analysis made shows that excretion of the first $50 \%$ of test food takes a similar course in the case of C. glareolus as it does in that of $M$. agrestis, and of $M$. arvalis as in that of $M$. nivalis. On the other hand, the course taken by the passage of the remainder of the indicator is similar in representatives of the genus Microtus, thus differing from C. glareolus. In general, however, the excretion of the green parts of plants is very similar in all the species examined (Fig. 1). 
The rate of passage of foodstuffs in Microtidae

Table 2.

Description of the course taken by excretion of the green parts of plants.

\begin{tabular}{|c|c|c|c|c|c|c|}
\hline \multirow{2}{*}{ No. } & \multirow{2}{*}{ Sex } & \multicolumn{4}{|c|}{ Excretion time of sta1ned food /hours and m1nutes/ } & \multirow{2}{*}{$\mathrm{R}$ values } \\
\hline & & 58 & $50 \pi$ & 905 & 1005 & \\
\hline 1 & 2 & 3 & 4 & 5 & 6 & 7 \\
\hline \multicolumn{7}{|c|}{ Microtus arvalis } \\
\hline 1 & \multirow[b]{6}{*}{ n } & 1.04 & 2.56 & 7.03 & 21.00 & 3.08 \\
\hline 2 & & 0.31 & 2.48 & 6.50 & 17.00 & 3.25 \\
\hline 3 & & 1.06 & 2.14 & 5.55 & 16.00 & 3.12 \\
\hline 4 & & 0.42 & 2.54 & 6.49 & 19.00 & 3.33 \\
\hline 5 & & 1.08 & 2.23 & 4.41 & 17.00 & 2.40 \\
\hline 6 & & 0.14 & 2.15 & 7.15 & 15.00 & 2.58 \\
\hline 7 & \multirow{2}{*}{$\begin{array}{l}\omega \\
H\end{array}$} & 1.04 & 2.45 & 6.00 & 18.00 & 3.18 \\
\hline 8 & & 1.13 & 4.07 & 7.36 & 17.00 & 4.13 \\
\hline 9 & \multirow{7}{*}{$\begin{array}{l}< \\
>\end{array}$} & 0.28 & 2.46 & 7.46 & 19.00 & 3.34 \\
\hline 10 & & 0.19 & 2.06 & 5.39 & 16.00 & 2.38 \\
\hline 11 & & 0.45 & 3.01 & 6.49 & 17.00 & 3.28 \\
\hline 12 & & 1.00 & 2.28 & 5.58 & 15.00 & 2.59 \\
\hline 13 & & 1.08 & 2.33 & 6.10 & 18.00 & 3.10 \\
\hline 14 & & 0.25 & 2.34 & 5.33 & 18.00 & 3.07 \\
\hline 15 & & 0.16 & 2.36 & 7.25 & 17.00 & 3.17 \\
\hline \multicolumn{2}{|c|}{$\bar{x}_{\delta \delta}$} & $0.46 \pm 0.11$ & $2.42 \pm 0.15$ & $6.30 \pm 0.29$ & $17.18 \pm 1.00$ & $3.15 \pm 0.09$ \\
\hline 16 & \multirow{5}{*}{ es } & 1.08 & 2.32 & 7.23 & 20.00 & 3.22 \\
\hline 17 & & 0.42 & 1.55 & 5.17 & 20.00 & 2.40 \\
\hline 18 & & 1.05 & 3.05 & 6.32 & 17.00 & 3.36 \\
\hline 19 & & 0.36 & 2.14 & 5.06 & 21.00 & 2. 39 \\
\hline 20 & & 0.31 & 2.38 & 6.09 & 16.00 & 2.59 \\
\hline 21 & $\infty 2$ & 0.28 & 2.23 & 7.32 & 20.00 & 3.14 \\
\hline 22 & \multirow{2}{*}{$\rightarrow$} & 0.26 & 2.34 & 7.28 & 17.00 & 3.22 \\
\hline 23 & & 1.10 & 2.58 & 6.56 & 18.00 & 3.50 \\
\hline 24 & \multirow[t]{2}{*}{$=$} & 1.02 & 2.33 & 5.01 & 19.00 & 2.58 \\
\hline 25 & & 0.20 & 2.55 & 5.55 & 16.00 & 3.19 \\
\hline 26 & \multirow{2}{*}{ is } & 1.13 & 2.43 & 7.01 & 17.00 & 1.57 \\
\hline 27 & & 0.42 & 2.27 & 6.35 & 16.00 & 3.07 \\
\hline 28. & \multirow{3}{*}{ 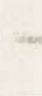 } & Lache 08 , & 3.12 & 7.10 & 17.00 & 3.50 \\
\hline $29^{\circ}$ & & 0.187 & 2.36 & 4.40 & 14.00 & 2.40 \\
\hline 30 & & 0.55 & 3.17 & 6.19 & 16.00 & 3.34 \\
\hline \multicolumn{2}{|c|}{$\bar{x}_{q q}$} & $0.44 \pm 0.14$ & $2.40 \pm 0.14$ & $6.20 \pm 0.16$ & $17.36 \pm 1.06$ & $3.08 \pm 0.19$ \\
\hline \multicolumn{2}{|c|}{$\bar{x} 6 \delta^{4}+9$} & $0.45 \pm 0.09$ & $2.41 \pm 0.09$ & $6.25 \pm 0.15$ & $17.27 \pm 0.43$ & $3.11 \pm 0.10$ \\
\hline \multicolumn{7}{|c|}{ M1crotus agrestis } \\
\hline 1 & \multirow{6}{*}{ os } & 0.26 & 1.58 & 4.47 & 14.00 & 2.25 \\
\hline 2 & & 0.32 & 2.16 & 5.56 & 15.00 & 3.07 \\
\hline 3 & & 1.03 & 1.58 & 4.33 & 15.00 & 2. 30 \\
\hline 4 & & 0.08 & 1.15 & 2.47 & 13.00 & 1.26 \\
\hline 5 & & 0.38 & 1.45 & 4.48 & 14.00 & 2.27 \\
\hline 6 & & 1.05 & 2.39 & 6.47 & 18.00 & 3.37 \\
\hline 7 & is & 0.07 & 1.09 & 4.19 & 19.00 & 1.41 \\
\hline 8 & $\rightarrow$ & 0.07 & 1.17 & 3.45 & 20.00 & 1.42 \\
\hline 9 & $<$ & 0.10 & 1.34 & 4.37 & 19.00 & 2.13 \\
\hline 10 & $x$ & 0.07 & 1.06 & 2.52 & 19.00 & 1.36 \\
\hline 11 & 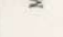 & 1.00 & 1.48 & 3.56 & 17.00 & 2.38 \\
\hline 12 & & 0.09 & 1.37 & 9.24 & 19.00 & 2.55 \\
\hline 13 & & 0.11 & 1.33 & 5.32 & 18.00 & 2.22 \\
\hline 14 & & 0.08 & 1.17 & 4.13 & 19.00 & 1.49 \\
\hline 15 & & 0.07 & 1.19 & 8.00 & 17.00 & 2.43 \\
\hline$\overline{\mathrm{x}}$ & & $0.24 \pm 0.12$ & $1.38 \pm 0.15$ & $5.02 \pm 0.66$ & $17.04 \pm 1.24$ & $2.21 \pm 0.25$ \\
\hline
\end{tabular}




\begin{tabular}{|c|c|c|c|c|c|c|}
\hline 1 & 2 & 3 & 4 & 5 & 6 & 7 \\
\hline 16 & \multirow{15}{*}{$\begin{array}{l}\infty \\
\infty \\
+ \\
\alpha \\
= \\
\infty\end{array}$} & 0.36 & 1.51 & 5.15 & 14.00 & 2.24 \\
\hline 17 & & 1.05 & 2.21 & 5.37 & 17.00 & 2.59 \\
\hline 18 & & 2.03 & 4.03 & 10.25 & 16.00 & 4.43 \\
\hline 19 & & 0.31 & 2.50 & 5.57 & 23.00 & 3.01 \\
\hline 20 & & 0.12 & 1.35 & 5.23 & 17.00 & 2.17 \\
\hline 21 & & 0.20 & 2.41 & 10.24 & 17.00 & 3.54 \\
\hline 22 & & 0.26 & 2.46 & 11.41 & 17.00 & 4. 31 \\
\hline 23 & & 0.30 & 2.17 & 8.01 & 18.00 & 3.34 \\
\hline 24 & & 0.08 & 1.28 & 4.08 & 17.00 & 1.55 \\
\hline 25 & & 1.16 & 5.00 & 9.35 & 17.00 & 4.57 \\
\hline 26 & & 0.38 & 2.06 & 9.24 & 18.00 & 4.05 \\
\hline 27 & & 0.06 & 1.06 & 5.27 & 17.00 & 2.10 \\
\hline 28 & & 0.26 & 1.47 & 7.56 & 18.00 & 3.07 \\
\hline 29 & & 0.25 & 2.10 & 9.00 & 18.00 & 3.26 \\
\hline 30 & & 0.07 & 1.09 & 2.48 & 19.00 & 1.21 \\
\hline \multicolumn{2}{|c|}{$\bar{x} q q$} & $0.35 \pm 0.18$ & $2.21 \pm 0.35$ & $7.17 \pm 1.40$ & $17.32 \pm 1.10$ & $3.16 \pm 0.32$ \\
\hline \multicolumn{2}{|c|}{$\bar{x} 8 \delta+\% q$} & $0.30 \pm 0.10$ & $1.59 \pm 0.18$ & $6.09 \pm 0.56$ & $17.18 \pm 0.51$ & $2.48 \pm 0.19$ \\
\hline \multicolumn{7}{|c|}{ Microtus n1valis } \\
\hline 1 & 0 & 0.45 & 3.24 & 10.00 & 20.00 & 4. 31 \\
\hline 2 & - & 0.17 & 2. 39 & 6.57 & 17.00 & 3.16 \\
\hline 3 & 0 & 0.36 & 1.58 & 5.25 & 16.00 & 2.27 \\
\hline 4 & o & 0.14 & 4. 11 & 6.50 & 20.00 & 4.16 \\
\hline 5 & $\circ$ & 1.54 & 5.16 & 7.57 & 16.00 & 5.22 \\
\hline 6 & $q$ & 0.31 & 2.36 & 10.28 & 17.00 & 3.50 \\
\hline 7 & $q$ & 0.29 & 3.47 & 7.20 & 19.00 & 3.54 \\
\hline & $\bar{x}$ & $0.41 \pm 0.28$ & $3.25 \pm 1.00$ & $7.51 \pm 1.40$ & $17.52 \pm 2.00$ & $3.57 \pm 0.50$ \\
\hline \multicolumn{7}{|c|}{ Clethrionomys glareolus } \\
\hline \multirow{9}{*}{8} & 0 & 1.04 & 1.44 & 14.53 & 19.00 & 4.53 \\
\hline & 0 & 0.08 & 1.28 & 5.20 & 18.00 & 2.18 \\
\hline & 0 & 0.12 & 1.44 & 8.08 & 15.00 & 2.48 \\
\hline & 0 & 0.10 & 1. 30 & 11.01 & 16.00 & 3.04 \\
\hline & 0 & 0.06 & 0.57 & 7.23 & 16.00 & 2.40 \\
\hline & 0 & 0.06 & 0.59 & 10.24 & 18.00 & 3.44 \\
\hline & $q$ & 0.06 & 0.57 & 5.20 & 21.00 & 1.58 \\
\hline & $q$ & 0.08 & 2.28 & 9.06 & 13.00 & 3.08 \\
\hline & \& & 0.06 & 1.05 & 11.31 & 19.00 & 3.23 \\
\hline & $\bar{x}$ & $0.14 \pm 0.14$ & $1.26 \pm 0.23$ & $9.14 \pm 2.23$ & $17.13 \pm 2.00$ & $3.06 \pm 0.40$ \\
\hline
\end{tabular}

2. Passage of seeds (Tables 4, 5; Fig. 2)

Stained particles appeared in the excretions of all the species during the first hour of the experiment. $50 \%$ of the test food is most rapidly excreted by $C$. glareolus $(6 \%$ /hour), and most slowly by $M$. arvalis $(4.29 \%)$ $M$. agrestis occupies an intermediate position ( $5 \% /$ hour), also $M$. nivalis $(5.40 \%$ /hour). The main mass of the test food also passes most rapidly through the alimentary tract of $C$. glareolus (16 hours), more slowly in the case of $M$. nivalis and $M$. agrestis (17.5 hours), and most slowly with M. arvalis (19 hours). After $75 \%$ of the indicator has been excreted, excretion gradually slows down and the final $10 \%$ was excreted by the 


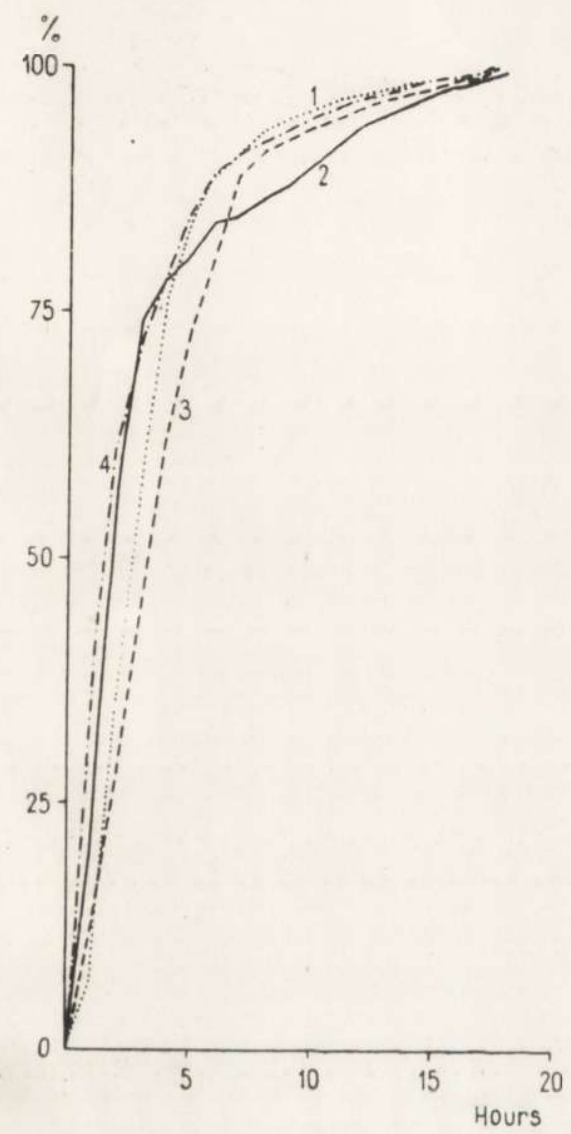

Fig. 1. Average course taken by excretion of the red stained green parts of plants.

$1-$ M. arvalis, $2-$ M. agrestis

$3-$ M. nivalis, $4-$ C. glareolus.

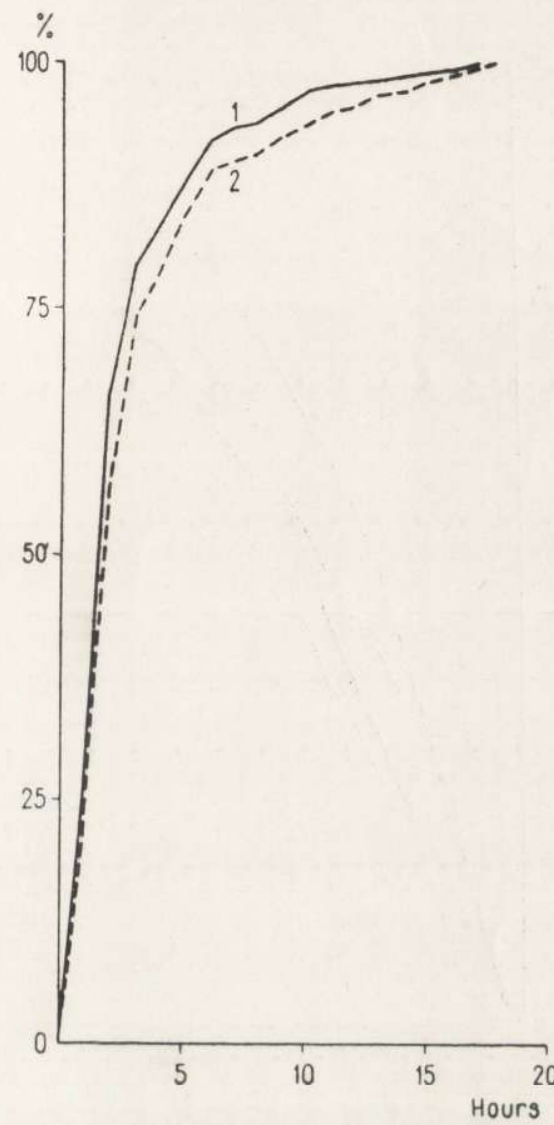

Fig. 3. Average course taken by excretion of indicator in males and females of Microtus agrestis fed on the green parts of plants.

1 - Males, 2 - Females.

rodents examined at a rate of $0.60 \%$ hour. The lowest $R$ value was obtained for C. glareolus, the highest for M. arvalis, and intermediate values for $M$. nivalis and M. agrestis. As can be seen, these values are distributed parallel to the rate of excretion of the test food. Comparison of the times of excretion of $5 \%, 50 \%, 90 \%$ and $100 \%$ of the indicator by C. glareolus and $M$. agrestis did not reveal statistically significant differences, such differences being obtained only from a comparison of $R$ values. Statistically significant differences are obtained when comparing all the above mentioned values calculated for $C$. glareolus with analogical data for $M$. arvalis. In analysing the process of excretion of wheat by $M$. arvalis and $M$. agrestis, significant differences were found only when 


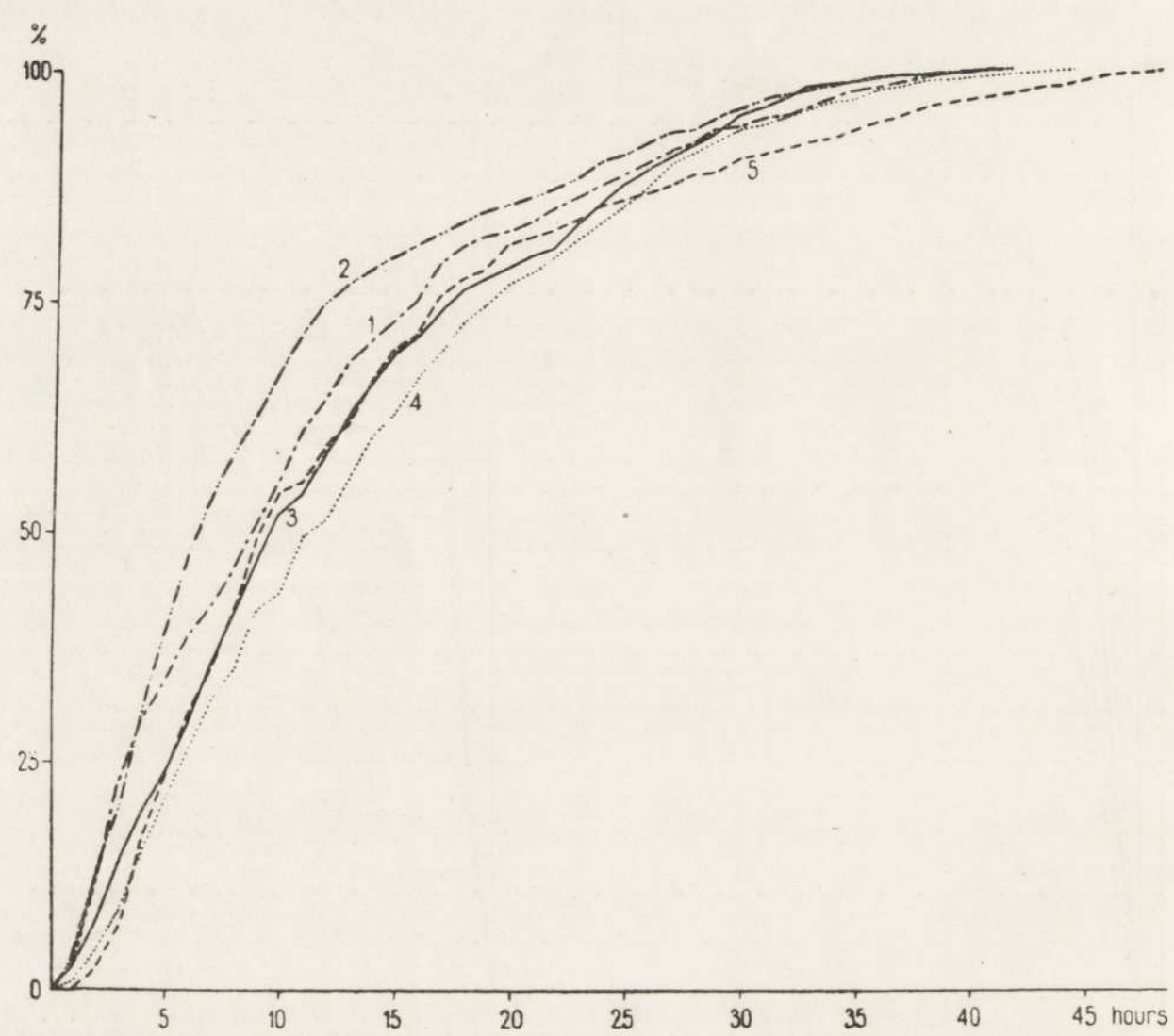

Fig. 2. Average course taken by excretion of red stained seeds.

1-C. glareolus - wheat; 2 - C. glareolus - acorns; $3-$ M. agrestis; 4-M. arvalis, 5 - nivalis - wheat.

the times of excretion of $50 \%$ and $100 \%$ of the indicator are compared The excretion of grain food by $M$. nivalis is similar to the course taken by this process in $M$. agrestis (statistically non-significant difference betweer. $R$ values and times of excretion of $5 \%$ and $50 \%$ of the test food). The excretion times of $50 \%$ and $100 \%$ of the indicator in $M$. arvalis and M. nivalis differ in a statistically different way.

The passage of acorns through the alimentary tract of $C$. glareolus (Table 5) is the same as the passage of wheat (differences statistically non-significant).

Despite the above-mentioned aifferences, the course taken by the passage of seeds in all the species of Microtidae examined is in general outlines similar (Fig. 2). 
Table 3.

Significance of differences between values describing the passage of the green parts of plants. The significance of differences between $\sigma^{*} \sigma^{*}$ and $q$ of these species is marked on the intersections of $M$. agrestis with $M$. agrestis and $M$. arvalis witi M arvalis.

\begin{tabular}{|c|c|c|c|c|c|c|c|c|c|c|c|c|c|c|c|c|}
\hline & & \multicolumn{5}{|c|}{ M. agrestis } & \multicolumn{5}{|c|}{ c. Glareolus } & \multicolumn{5}{|c|}{ u. srvalis } \\
\hline & & 56 & $50 \pi$ & 906 & 1008 & R & 58 & 506 & 905 & $100 \%$ & 3 & $5 \%$ & $50 \%$ & 908 & $100 \pi$ & R \\
\hline \multirow{5}{*}{ 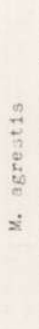 } & $5 x$ & - & & & & & - & & & & & & & & & \\
\hline & $50 \pi$ & & - & & & & & - & & & & & & & & \\
\hline & 905 & & & + & & & & & + & & & & & & & \\
\hline & $100 \pi$ & & & & - & & & & & - & & & & & & \\
\hline & a & & & & & + & & & & & - & & & & & \\
\hline \multirow{5}{*}{$\begin{array}{l}\frac{5}{7} \\
\frac{7}{2} \\
\text { है } \\
\dot{z}\end{array}$} & 58 & - & & & & & + & & & & & - & & T & & \\
\hline & 504 & & + & & & & & + & & & & & + & & & \\
\hline & $90 \pi$ & & & - & & & & & - & & & & & + & & \\
\hline & $100 \pi$ & & & & - & & & & & - & & & & & - & \\
\hline & $\mathrm{R}$ & & & & & + & & & & & - & & & & & + \\
\hline \multirow{5}{*}{ 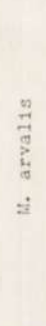 } & 54 & + & & & & & + & & & & & - & & & & \\
\hline & $50 \pi$ & & + & & & & & + & & & & & - & & & \\
\hline & $20 \pi$ & & & - & & & & & + & & & & & - & & \\
\hline & 1004 & & & & - & & & & & - & & & & & - & \\
\hline & R & & & & & + & & & & & - & & & & & - \\
\hline
\end{tabular}

3. Comparison of the rate of passage of the green parts of plants with the rate of passage of seeds

The process of excreting both kinds of food always began during the first hour following the time at which the animals were given the test food. The main mass of the stained green parts of plants passes $4-5$ times more quickly through the alimentary tract than the main mass of the stained seed food. The rate of excretion of the remainder of the indicator 
Table 4.

Description of the course taken by excretion of wheat grains.

\begin{tabular}{|c|c|c|c|c|c|c|}
\hline \multirow{2}{*}{ No. } & \multirow{2}{*}{ Sex } & \multicolumn{4}{|c|}{ Excretion time of stained food /hours and tainutes/ } & \multirow{2}{*}{ R values } \\
\hline & & 5 & 50 . & 905 & 1005 & \\
\hline 1 & 2 & 3 & 4 & 5 & 6 & 7 \\
\hline \multicolumn{7}{|c|}{ Clethrionomys Blareolus } \\
\hline 1 & \multirow{6}{*}{ s } & 1.00 & 4.49 & 22.41 & 37.00 & 8.23 \\
\hline 2 & & 1.36 & 8. 36 & 20.13 & 39.00 & 9.39 \\
\hline 3 & & 1.22 & 8.36 & 14.19 & 39.00 & 9.21 \\
\hline 4 & & 1.32 & 6.46 & 24.23 & 39.00 & 8.57 \\
\hline 5 & & 1.23 & 8.30 & 24.42 & 41.00 & 10.24 \\
\hline 6 & & 3.06 & 12.19 & 29.47 & 42.00 & 14.27 \\
\hline 7 & \multirow{2}{*}{$\begin{array}{l}\text { frl } \\
H\end{array}$} & 1.15 & 9.34 & 23.43 & 43.00 & 10.53 \\
\hline 8 & & 1.17 & 17.41 & 33.42 & 43.00 & 16.07 \\
\hline 9 & \multirow{7}{*}{$\begin{array}{l}< \\
\leq\end{array}$} & 0.47 & 12.07 & 23.30 & 40.00 & 12.00 \\
\hline 10 & & 0.33 & 3.22 & 28.13 & 42.00 & 8.35 \\
\hline 11 & & 1.18 & 8.40 & 25.54 & 38.00 & 10.51 \\
\hline 12 & & 1.12 & 8.44 & 20.03 & 41.00 & 10. 38 \\
\hline 13 & & 3.05 & 6.49 & 31.04 & 42.00 & 12.21 \\
\hline 14 & & 0.57 & 7.58 & 21.44 & 40.00 & 10.22 \\
\hline 15 & & 1.42 & 8.40 & 25.17 & 40.00 & 10.22 \\
\hline \multicolumn{2}{|c|}{$\bar{x}_{\delta \delta}$} & $1.28 \pm 0.25$ & $8.53 \pm 1.53$ & $24.39 \pm 2.35$ & $40.24 \pm 1.00$ & $10.53 \pm 1.12$ \\
\hline 16 & \multirow{6}{*}{$\infty$} & 1.07 & 2.21 & 17.59 & 38.00 & 5.52 \\
\hline 17 & & 5.07 & 12.34 & 35.53 & 41.00 & 15.58 \\
\hline 18 & & 6.26 & 13.11 & 27.59 & 40.00 & 15.30 \\
\hline 19 & & 1.52 & 5.55 & 25.31 & 41.00 & 10.00 \\
\hline 20 & & 2.17 & 8.48 & 23.30 & 40.00 & 10.50 \\
\hline 21 & & 1.51 & 5.37 & 16.12 & 45.00 & 9.52 \\
\hline 22 & $\mapsto$ & 1.10 & 9.48 & 27.08 & $4 \pi .00$ & 11.25 \\
\hline 23 & \multirow{2}{*}{$\begin{array}{l}4 \\
=\end{array}$} & 1.19 & 12.00 & 28.08 & 38.00 & 13.45 \\
\hline 24 & & 1.32 & 5.34 & 25.41 & 38.00 & 9.39 \\
\hline 25 & \multirow{2}{*}{$\omega$} & 0.27 & 10.11 & 26.41 & 41.00 & 10.44 \\
\hline 26 & & 1. 38 & 12.05 & 22.43 & 39.00 & 12.34 \\
\hline 27 & \multirow[t]{4}{*}{ in } & 0.49 & 6.42 & 26.48 & 41.00 & 9.57 \\
\hline 28 & & 1.06 & 7.50 & 35.25 & 43.00 & 13.52 \\
\hline 29 & & 0.37 & 4.32 & 22.33 & 38.00 & 9.23 \\
\hline 30 & & 2. 32 & 7.02 & 20.32 & 39.00 & 9.13 \\
\hline \multicolumn{2}{|c|}{$\overline{\mathrm{X}}_{+\%}$} & $1.59 \pm 0.56$ & $8.15 \pm 1.53$ & $25.31 \pm 3.02$ & $40.24 \pm 1.18$ & $11.14 \pm 1.30$ \\
\hline \multicolumn{2}{|c|}{$\bar{x}$ od +9} & $1.43 \pm 0.28$ & $8.34 \pm 1.14$ & $25.05 \pm 1.51$ & $40.24 \pm 0.46$ & $11.03 \pm 0.54$ \\
\hline \multicolumn{7}{|c|}{ M1crotus agrestis } \\
\hline 1 & \multirow{6}{*}{ os } & 4.17 & 8.28 & 27.33 & 42.00 & 13.20 \\
\hline 2 & & 2. 35 & 10.46 & 25.48 & 40.00 & 13.28 \\
\hline 3 & & 9.12 & 12.06 & 13.57 & 41.00 & 12.34 \\
\hline 4 & & 2.25 & 8.53 & 28.07 & 38.00 & 11.25 \\
\hline 5 & & 3.12 & 9.08 & 29.00 & 37.00 & 13.10 \\
\hline 6 & & 2.29 & 11.41 & 32.31 & 40.00 & 14.57 \\
\hline 7 & \multirow{2}{*}{$\begin{array}{l}\infty \\
\Delta\end{array}$} & 1.44 & 12.23 & 28.43 & 45.00 & 14.15 \\
\hline 8 & & 2.22 & 15.25 & 30.12 & 39.00 & 15.45 \\
\hline 9 & $<$ & 1.31 & 16.41 & 29.57 & 43.00 & 15.46 \\
\hline 10 & $\geq$ & 1.14 & 9.33 & 24.35 & 40.00 & 11.49 \\
\hline 11 & & 1.17 & 9.30 & 26.42 & 45.00 & 12.38 \\
\hline 12 & & 1.19 & 7.51 & 23.26 & 44.00 & 10.14 \\
\hline 13 & & 1.22 & 11.06 & 25.04 & 43.00 & 11.22 \\
\hline 14 & & 2.26 & 9.02 & 24.00 & 39.00 & 12.09 \\
\hline 15 & & 2.29 & 13.44 & 26.10 & 42.00 & 14.40 \\
\hline & & $2.40 \pm 1.06$ & $11.05 \pm 1.28$ & $26.23 \pm 3.27$ & $41.12 \pm 1.25$ & $13.10 \pm 0.56$ \\
\hline
\end{tabular}




\begin{tabular}{|c|c|c|c|c|c|c|}
\hline 1 & 2 & 3 & 4 & 5 & 6 & 7 \\
\hline 16 & \multirow{5}{*}{ on } & 0.45 & 5.42 & 24.29 & 41.00 & 8.30 \\
\hline 17 & & 1.24 & 9.41 & 30.27 & 40.00 & 12.51 \\
\hline 18 & & 0.28 & 9.48 & 25.52 & 40.00 & 10.50 \\
\hline 19 & & 1.13 & 9.19 & 27.52 & 39.00 & 10.56 \\
\hline 20 & & 0.30 & 7.06 & 21.33 & 41.00 & 8.45 \\
\hline 21 & $\infty$ & 5.24 & 12.38 & 28.48 & 38.00 & 19.22 \\
\hline 22 & \multirow{2}{*}{$\rightarrow$} & 0.59 & 9.46 & 23. 57 & 40.00 & 12.27 \\
\hline 23 & & 3.15 & 8.40 & 24.13 & 37.00 & 11.51 \\
\hline 24 & \multirow[t]{3}{*}{2} & 1.34 & 9.12 & 28.22 & 43.00 & 12.03 \\
\hline 25 & & 1.33 & 9.23 & 26.00 & 42.00 & 11.49 \\
\hline 26 & & 0.26 & 8.19 & 24.00 & 42.00 & 10.52 \\
\hline 27 & \multirow[t]{4}{*}{$\infty$} & 0.38 & 7.03 & 26.47 & 38.00 & 10.31 \\
\hline 28 & & 2.23 & 6.19 & 25.53 & 41.00 & 10.12 \\
\hline 29 & & 1.54 & 11.18 & 26.13 & 39.00 & 12.57 \\
\hline 30 & & 0.36 & 8.12 & 26.00 & 40.00 & 11.13 \\
\hline \multicolumn{2}{|c|}{$\bar{x}_{q q}$} & $1.32 \pm 0.45$ & $8.50 \pm 1.00$ & $26.02 \pm 1.07$ & $40.04 \pm 0.49$ & $11.25 \pm 0.55$ \\
\hline \multicolumn{2}{|c|}{$\bar{x} \delta f^{+} q q$} & $2.06 \pm 0.38$ & $9.58 \pm 0.54$ & $26.12 \pm 1.14$ & $40.38 \pm 0.46$ & $12.17 \pm 0.44$ \\
\hline \multicolumn{7}{|c|}{ M1crotus arval1s } \\
\hline 1 & \multirow{15}{*}{$\begin{array}{l}4 \\
= \\
=\end{array}$} & 2.36 & 10.22 & 29.35 & 45.00 & 13.27 \\
\hline 2 & & 2.07 & 10.35 & 23.45 & 51.00 & 12.57 \\
\hline 3 & & 1.20 & 10.56 & 27.15 & 46.00 & 12.40 \\
\hline 4 & & 3.00 & 10.26 & 27.04 & 44.00 & 13.03 \\
\hline 5 & & 1.15 & 7.25 & 27.04 & 51.00 & 11.29 \\
\hline 6 & & 2.06 & 8.30 & 26.06 & 42.00 & 11.22 \\
\hline 7 & & 1.34 & 12.33 & 27.47 & 49.00 & 14.19 \\
\hline 8 & & 3.18 & 12.35 & 27.20 & 42.00 & 13.39 \\
\hline 9 & & 2.19 & 14.16 & 20.43 & 46.00 & 14.04 \\
\hline 10 & & 2.36 & 10.20 & 27.54 & 46.00 & 13.53 \\
\hline 11 & & 4.23 & 14.35 & 28.49 & 39.00 & 16.56 \\
\hline 12 & & 1.41 & 11.40 & 24.49 & 45.00 & 12.17 \\
\hline 13 & & 2.03 & 15.29 & 30.10 & 44.00 & 15.55 \\
\hline 14 & & 8.27 & 15.44 & 19.52 & 42.00 & 15.34 \\
\hline 15 & & 2. 30 & 10.16 & 27.12 & 42.00 & 12.42 \\
\hline \multicolumn{2}{|c|}{$\bar{x}_{\delta \gamma}$} & $2.45 \pm 1.00$ & $11.43 \pm 1.22$ & $27.02 \pm 1.20$ & $44.56 \pm 1.54$ & $13.37 \pm 0.54$ \\
\hline 16 & \multirow{6}{*}{$\infty$} & 2.03 & 8.52 & 33.08 & 49.00 & 13.24 \\
\hline 17 & & 2.09 & 12.23 & 26.21 & 46.00 & 12.27 \\
\hline 18 & & 1.28 & 12.51 & 28.10 & 47.00 & 13.25 \\
\hline 19 & & 3.18 & 10.23 & 26.38 & 45.00 & 12.53 \\
\hline 20 & & 2.16 & 10.19 & 26.07 & 46.00 & 12.32 \\
\hline 21 & & 2. 11 & 10.57 & 25.03 & 42.00 & 12.27 \\
\hline 22 & $\rightarrow$ & 3.11 & 8.23 & 24.34 & 43.00 & 11.04 \\
\hline 23 & $<$ & 1.15 & 6.03 & 25.30 & 37.00 & 10.16 \\
\hline 24 & \multirow{2}{*}{$z$} & 2.25 & 13.36 & 30.55 & 44.00 & 15.45 \\
\hline 25 & & 1.33 & 11.43 & 26.00 & 40.00 & 13.04 \\
\hline 26 & \multirow[t]{5}{*}{ a. } & 2.26 & 10.43 & 29.34 & 46.00 & 14.00 \\
\hline 27 & & 2. 51 & 12.44 & 29.51 & 45.00 & 15.25 \\
\hline 28 & & 1.52 & 11.46 & 33.00 & 44.00 & 14.53 \\
\hline 29 & & 2.23 & 13.02 & 25.50 & 43.00 & 13.56 \\
\hline 30 & & 3.17 & 9.56 & 27.00 & 44.00 & 13.46 \\
\hline \multicolumn{2}{|c|}{$\overline{\mathbf{x}}_{q}$} & $2.20 \pm 0.20$ & $10.55 \pm 1.08$ & $27.50 \pm 1.33$ & $44.04 \pm 1.38$ & $13.17 \pm 0.51$ \\
\hline \multicolumn{2}{|c|}{$\bar{x} \not f+q q$} & $2.32 \pm 0.30$ & $11.19 \pm 0.50$ & $27.26 \pm 1.00$ & $44.30 \pm 1.12$ & $13.27 \pm 0.34$ \\
\hline & & & M1crotus & n1valis & & \\
\hline 1 & d & 3.09 & 7.11 & 31.48 & 47.00 & 12.15 \\
\hline 2 & $\sigma$ & 2.50 & 9.00 & 27.47 & 46.00 & 13.04 \\
\hline 3 & $\sigma$ & 2.22 & 12.46 & 37.04 & 53.00 & 15.07 \\
\hline 4 & $\sigma^{\prime}$ & 3.23 & 7.45 & 23.48 & 45.00 & 11.05 \\
\hline 5 & $q$ & 1.33 & 7.50 & 31.38 & 46.00 & 12.15 \\
\hline 6 & $q$ & 2.28 & 9.58 & 29.30 & 53.00 & 13.13 \\
\hline $\bar{x}$ & & $2.37 \pm 0.43$ & $9.05 \pm 2.53$ & $30.16 \pm 4.39$ & $48.20 \pm 4.18$ & $12.50 \pm 1.23$ \\
\hline
\end{tabular}


in both cases increases gradually and becomes similar to each other in the final phase. The end of excretion of the green food takes place $2.5-3$ times earlier than in the case of seed food. It is obvious from the above that after the main mass of the indicator has been excreted, the rate of excreting the green parts of plants decreases far more considerably than the rate of excreting seeds, as can be seen by comparing the curves of excretion (Fig. 1,2). These differences are expressed in the $R$ values, which are about four times greater in the case of seeds, as compared with the $R$ value for the passage of green food. The main mass of the indicator of the passage of both kinds of food is most rapidly excreted by $C$. glareolus, more slowly by $M$. agrestis, and most slowly by $M$. arvalis. The course taken by the excretion of the green parts of plants in M. nivalis is similar to this process in M. arvalis, while excretion of the remainder of

Table 5.

Description of the course taken by excretion of acorns in C. glareolus.

\begin{tabular}{|c|c|c|c|c|c|c|}
\hline \multirow{2}{*}{ No. } & \multirow{2}{*}{$\operatorname{Sex}$} & \multicolumn{4}{|c|}{ Excretion time of sta1ned food /hours and minutes/ } & \multirow{2}{*}{ R values } \\
\hline & & $5 \%$ & $50 \%$ & $90 \%$ & $100 \%$ & \\
\hline 1 & $\sigma^{\prime}$ & 2.33 & 8.42 & 25.55 & 43.00 & 11.35 \\
\hline 2 & $\sigma^{\prime}$ & 2.25 & 9.54 & 23.18 & 41.00 & 11.04 \\
\hline 3 & o & 1.08 & 4.18 & 26.03 & 40.00 & 8.33 \\
\hline 4 & $\sigma$ & 3.10 & 9.38 & 26.56 & 45.00 & 12.59 \\
\hline 5 & $q$ & 1.17 & 6.45 & 23.03 & 41.00 & 9.31 \\
\hline 6 & $q$ & 0.27 & 4.05 & 19.33 & 41.00 & 6.45 \\
\hline 7 & $q$ & 1.03 & 5.16 & 15.30 & 44.00 & 6.57 \\
\hline \multicolumn{2}{|c|}{$\bar{x}$} & $1.43 \pm 0.56$ & $6.57 \pm 3.12$ & $22.49 \pm 4.16$ & $42.08 \pm 2.00$ & $9.38 \pm 2.11$ \\
\hline
\end{tabular}

the wheat takes place in the case of this species in a similar way to that in $M$. agrestis.

The data obtained show that despite the occurrence of differences between the species of Microtidae examined in the rate of passage of food, this rate depends primarily on the kind of food, and to a far lesser degree on the species of rodents.

\section{Sex dimorphism in the rate of passage of foodstuffs}

The passage of seeds follows a uniform course in both sexes of C. glareolus, M. agrestis and $M$. arvalis. Comparison of $R$ values and excretion times of $5 \%, 50 \%, 90 \%$ and $100 \%$ of the indicator reveals statistically non-significant differences. The rate of passage of the green parts of plants through the alimenary tract of males and females of M. arvalis is also uniform (statistically non-significant differences). This process differs slightly in both sexes in the case of M. agrestis, for which a sta- 
tistically significant difference was obtained when comparing the excretion time of $90 \%$ of the stained food, and also when comparing $R$ values (Fig. 3).

\section{DISCUSSION}

Experiments made on species feeding on different kinds of food and possessing differently built digestive systems made it possible to establish, in the case of the Microtidae examined, the degree to which the rate of passage of foodstuffs is dependent on the kind of food and species of animal.

The rate of excretion of the main mass of the test food by $C$. glareolus, more rapid than in the other species discussed, is conditioned by the long small intestine and the relatively short caecum and large intestine. The cause of reduction in the rapidity of excretion of the final amounts of the indicator in the rodents examined was the retention of the stained food in their caecum and large intestine. A similar decrease in the excretion rate in the final phase of excretion was observed in nutria also (Gill \& Bi e guszewski, 1960). Velits h ko \& Moke jeva (1949) found that food remains longest in the posterior section of the rodents' intestines. Pra vdi n a (1958) showed that the green parts of plants pass more rapidly through the alimentary tract of Rodentia than seed food. Our data agree with these observations, but more exact comparison of results is impossible on account of the different investigation methods used by the above-mentioned authors.

The differences between the species of Microtidae examined in the rate of passage of the same kind of food are slight. When comparing $R$ values and excretion times of $5 \%, 50 \%, 90 \%$ and $100 \%$ of the indicator of any two of the species examined, statistically significant results were never obtained simultaneously in all of the cases.

In all the Microtidae examined the curves describing the passage of the same kind of food are very similar, while in the same species the passage of different kinds of food takes an entirely different course. This proves that in the Microtidae examined the rate of passage of foodstuffs depends on the kind of food, and only to a very slight degree on the species of animal.

The statistically significant differences obtained between males and females of $M$. agrestis when comparing $R$ values and excretion time of $90 \%$ of the remainder of the green parts of plants are probably fortuitous and are not of any importance. It may therefore be said that there is no sex dimorphism in the rate of passage of food through the alimentary system of the Microtidae examined. 


\section{SUMMARY}

Passage of the green parts of plants and of seeds through the alimentary tract was examined in 179 individuals belonging to four species of the family Microtidac. The method worked out by Castle (1956) and modified by Gill (1957) was use] in the experiments. The part of the food stained with basic fuchsin was used as a) indicator.

The rate of passage of food through the digestive system in the species examineu depends on the kind of food. Passage of green food takes place 2.5-3.0 times faster than the passage of seeds.

The first stained food appears in the excrement within the first hour of all the experiments. The main mass of the indicator of the passage of green food was excreted after 3-6 hours, and the main mass of stained seeds after 16-19 hours, C. glareolus excreting most rapidly and $M$. arvalis most slowly in both cases. A considerable decrease in the rate of passage of the final $10 \%$ of the indicator was observed in all the experiments.

The course taken by the passage of the same kind of food is similar in generai outline in all the species examined. Statistically significant differences were, however, found when comparing certain $R$ values and excretion times of $5 \%, 50 \%, 90 \%$ and $100 \%$ of the indicator. The greatest similarity in the passage of the green parts of plants occurs in C. glareolus and M. agrestis, and in M. arvalis and M. nivalis The passage of seeds takes place similarly in $M$. agrestis and $M$. nivalis. Statistically significant differences in the rate of passage of wheat grains and acorns in C. glareolus were not obtained.

The rate of passage of foodstuffs through the alimentary system of the Microtidae examined does not depend on sex. Statistically significant differences were obtained only for $R$ values and the excretion time of $90 \%$ of the indicator for males and females of $M$. agrestis fed on the green parts of plants.

Acknowledgments: We should like to record our gratitude to the late Professor Dr. August Dehnel, and to Dr. Zdzisław Pucek, for the assistance given in preparing this paper.

\section{REFERENCES}

1. B a l ch, C. C., 1950: Factors affecting the utilization of food by dairy cows. 1 . The rate of passage through the digestive tract. Brit. J. Nutr., 4: $361-388$. London.

2. B a shenina, N. V., 1962: Ekologija obyknovennoj polevki i nekotorye čerty ee geografičeskoj izmenčivosti. Izd. Mosk. Univ.: 1-308. Moskva.

3. B a u m a n n, R., 1949: Die freilebenden Säugetiere der Schweiz. Verl. Hans Huber: $1-492+$ XVI. Bern.

4. Castle, E. J., 1956: The rate of passage of foodstuffs through the alimentary tract of the goat. Brit. J. Nutr., 10: 15-23. London.

5. C a st le, E. J. \& C a s t l e, M. E., 1956: The rate of passage of food through the alimentary tract of pigs. J. Agric. Sci., 47: 196-204. London.

6. Castle, E. J. \& C a stle, M. E., 1957: Further studies on the rate of passiage of food through the alimentary tract of pigs. J. Agric. Sci., 49: 106-115, Londion. 
7. Gi11, J., 1957: Próby oznaczania szybkości przechodzenia treści przez przewód pokarmowy dzikich przeżuwaczy (jeleń - Cervus elaphus L., daniel - Damu dama L. i lama - Lama lama L.). Acta physiol. polon., 8, 3: 336-338. Warszawa.

8. Gill, J., 1959: Die Durchgangszeiten der Nahrung durch den Verdauungskanal des Elches, Alces alces (L.). Papers of the Fourth Congress of the International Union of Game Biologists in Arnhem (Holland).

9. Gill, J., 1960: Szybkość przechodzenia treści przez przewód pokarmowy słonia indyjskiego (Elephas maximus L.) w warunkach ogrodu zoologicznego. Acta physiol. polon., 11, 2: 277-287. Warszawa.

10. Gill, J. \& Bieguszewski, H., 1960: Die Durchgangszeiten der Nahrung durch den Verdauungskanal der Nutria, Myocastor coypus M o li n a, 1782. Acta theriol. 4, 2: 11-26. Białowieża.

11. Golenistschev, N. N., 1952: Vlijanie nedostatočnogo pitanija na razvitie obyknovennoj polevki. Tr. Vsesojuzn. In-ta Zašč. Rast., 4: 133-142. Leningrad.

12. Golley, F. B., 1960: Anatomy of the digestive tract of Microtus. J. Mammal., 41, 1: 89-99. Lawrance.

13. Górecki, A. \& G ęb czyńs ka, Z., 1962: Food conditions for small rodents in a deciduous forest. Acta theriol., 6, 10: 275-295. Białowieża.

14. Harder, W., 1950: Zur Morphologie und Physiologie des Blinddarmes der Nagetiere. Verhandl. d. Deutsch. Zoolog. Akad. Verl. Geest u. Portig K.-G.: 95-109. Leipzig.

15. Honigmann, H., 1936: Studies on nutrition of mammals. Part I Proc. zool. Soc. Lond., 10: 517-530. London.

16. Horszczaruk, F. 1962: Wpływ zróżnicowanego poziomu włókna surowego w dawkach na procesy trawienne u świń. II. Długość i pojemność przewodu pokarmowego oraz czas przechodzenia treści pokarmowej. Rocz. Nauk Roln. Ser. B., $80,2: 115-125$. Warszawa.

17. Ka ga ntsova, R. M., 1954: Vlijanie uslovij teploobmena i pitanija na razvitie obyknovennoj i obščstvennoj polevok. III ekol. konf. Tez. dokl., 3: 62-66. Kiev.

18. Kowa lski, K., 1964: Gryzonie. Klucze do oznaczania kręgowców Poiski. V. Ssaki - Mammalia. Państw. Wyd. Nauk. Warszawa-Kraków (in print.).

19. Koźniewski, S., 1961: Oznaczanie czasu przechodzenia treści pokarmowej u koni z trwałymi przetokami jelita ślepego. Med. wet., 17, 4: 236-240. Warszawa.

20. Kulajeva, M. T., 1958: Materialy po ekologičeskoj morfologii ryžih polevok. Izv. Kazan. Fil. Ak. Nauk SSSR. Ser. biol. nauk, 6: 7-25. Kazan'.

21. Lenkeit, W., 1931: Die Durchgangszeiten der Nahrung und der Verlauf der Ausscheiden durch den Verdauungskanal der Schweinen. Arch. f. Tierernähr. u. Tierzucht, 5: 376-385. Berlin.

22. Lenkeit, W. \& Ha beck, R., 1950: Zur Bestimmung der Durchgangszeiten den Verdauungskanal verschiedener Tiere. Arch. f. Tierernähr. u. Tierzucht, 2: 517-530. Berlin.

23. M a ngold, E., 1950: Die Verdauung bei den Nutztieren. Akademie Verl.: 1 1-108. Berlin.

24. M a n g o ld, E. \& Behm, G., 1954: Der Einfluss des Rohfasergehaltes im Futter auf die Durchgangszeiten beim Schwein. Arch. Tierernähr., 5, 10. Berlin. 
25. Miller, R. S., 1954: Food habits of the wood-mouse, Apodemus sylvaticus (L in ne, 1758) and the bank vole, Clethrionomys glareolus (S ch re be r, 1780) in the Wytham Woods, Berkshire. Säugetierkdl. Mitt,, 2, 3: 100--114. Stuttgart.

26. N a u mov, N. P., 1948: Očerki sravnitel'noj ekologii myševidnyh gryzunov. Izd. Ak. Nauk SSSR.: 1-204. Moskva-Leningrad.

27. N a s a r ova, I. V., 1958: Ekologo-morfologičeskij očerk seryh polevok Volžsko-kamskogo kraja. Izv. Kazan. Fil. Ak. Nauk SSSR. Ser. biol. nauk, 6: 27-40. Kazan'.

28. Neseni, R., Le cht, M. \& S chewen, B., 1955: Uber die Durchgangszeiten des Futters beim Silberfuchs. Arch. f. Tierernähr., 5, 26: 32-44. Berlin.

29. Neseni, R. \& Piatkowski, B., 1958: Die Durchgangszeiten des Futters beim Nerz. Arch. f. Tierernähr., 8, 4. Berlin.

30. O gne v, S. I., 1950: Zveri SSSR i priležaščih stran. Izd. Ak. Nauk SSSR, t. 7: 1-706. Moskva-Leningrad.

31. Pravdina, L. M., 1958: O dlitel'nosti prebyvanija korma v piščevaritel'nom trakte obyknovennoj i obščestvennoj polevok i beloj myši. Sb. "Biol. osnovy borby s gryzunami”. Tr. Vsesojuzn. In-ta Zašč. Rast., 12: 138-141. Moskva.

32. Sławiński, T., Bednarz, M. \& Sła woń, J .,1962: Wstępne badania nad transportem treści pokarmowej u lisów srebrzystych (Vulpes vulpes L.) i u lisów niebieskich (Alopex lagopus L.). Roczn. Nauk Roln. Ser. B. - Zootechn., 80, 2: 187-198. Warszawa.

33. Sła wiński, T., Sła woń, J. \& Bednarz, M., 1962: Transport treści pokarmowej u norek (Mustella vison Sch re b.). Roczn. Nauk Roln. Ser. B - Zoo. techn., 80, 2: 169-186. Warszawa.

34. Schwarz, S. S., 1960: Nekotorye zakonomernosti ekologičeskoj obuslovlennosti interernyh osobennostej nazemnyh pozvonočnyh životnyh. Sb. „Problemy flory i fauny Urala". Tr. In-ta Biol. Izd. Ak. Nauk SSSR., 14: 113-177. Sverdlovsk.

35. V elits h k o, M. A., 1956: O primenenii rentgenoskopičeskogo metoda issledovanija skorosti prohoždenija pišči u gryzunov. Uč. Zap. Leningr. Ped. In-ta. F-t estestvozn., 19, 5: 161-168. Leningrad.

36. Velitshko, M. A. \& Mokejeva, T. M., 1949: O nekotoryh harakternyh osobennostjah stroenija i funkcii kišečnika gryzunov. Tr. Vsesojuzn. In-ta Zašč. Rast., 2: 157-161. Moskva-Leningrad.

37. Vorontsov, N. N., 1961: Ekologičeskie i nekotorye morfologičeskie osobennosti ryžih polevok (Clethricnomys T i l.) evropejskogo severo-vostoka. Sb. „Mor fologija i ekologija pozvonočnyh životnyh". Izd. Ak. Nauk SSSR.: 101-156. Moskva-Leningrad.

38. Vor onts ov, N. N., 1962: The ways of food specialization and evolution of the alimentary system in Muroidea. Symp. theriol. Brno 1960. Nakl. Cesk. Ak Ved.: $360-377$. Praha.

39. Vor on ov, A. G., 1954: Osobennosti kormovogo raciona nekotoryh gryzunov. Zool. Żurn., 33, 1: 184-198. Moskva.

40. Zimmermann, K., 1959: Taschenbuch unserer wildlebenden Säugetiere Urania Verl.: 1-169. Leipzig-Jena, 


\section{STRESZCZENIE}

Transport wegetatywnych części roślin i nasion przebadano u 179 osobników należących do czterech gatunków z rodziny Microtidae (Tabela 1). W eksperymentach zastosowano metodę opracowaną przez Castle (1956) i zmodyfikowaną przez Gill a (1957). Jako wskaźnika użyto części pożywienia zabarwionej fuksyną zasadową. Pokarm testowy podawano zwierzętom raz dziennie. Barwne resztki liczono w pięciu polach widzenia mikroskopu w próbkach kału zbieranych w odstępach jednogodzinnych i odpowiednio rozcieńczanych. Następnie obliczono procent barw. nych resztek wydalonych w poszczególnych godzinach doświadczeń i wykreślon。 krzywe w/g Balcha (1950). Wyznaczono także wartości $R$ (Castle, 1956), obliczono czasy wydalania $5 \%, 50 \%, 90 \%$ i $100 \%$ pożywienia testowego i otrzymane wyniki poddano analizie statystycznej (test t-Studenta).

Szybkość przechodzenia pokarmu przez przewód trawienny badanych gatunków zależy od rodzaju pożywienia. Transport pokarmu zielonego odbywa się z szybkością 2,5-3 razy większą od transportu nasion.

Pierwsze barwne resztki pojawiały się w kale już w pierwszej godzinie wszyst. kich eksperymentów. Główna masa wskaźnika transportu pokarmu zielonego zostaje usunięta po 3-6 godzinach, a główna masa barwionych nasion po 16-19 godzinach, przy czym najszybciej w obu wypadkach wydala ją C. glareolus, a najwolniej M. arvalis (Ryc. 1, 2). We wszystkich doświadczeniach zaobserwowano duży spadek prędkości transportu ostatnich $10 \%$ wskaźnika. Koniec wydalania wegetatywnych części roślin następował po $17-18$ godzinach, a nasion po $40-48$ godzinach (Tabela $2,4,5)$.

Przebieg transportu tego samego rodzaju pokarmu jest w ogólnych zarysach podobny u wszystkich badanych gatunków (Ryc. 1, 2). Stwierdzono jednak różnice statystycznie istotne przy porównaniu niektórych wartości $R$ oraz czasów wydalenia $5 \%, 50 \%, 90 \%$ i $100 \%$ wskaźnika. Największe podobieństwo w transporcie wegetatywnych części roślin występuje u $C$. glareolus i $M$. agrestis oraz u $M$. arvalis i $M$. nivalis (Tabela 3). Transport nasion u $M$. agrestis przebiega podobnie jak u $M$. nivalis. Nie otrzymano różnic istotnych statystycznie w tempie przechodzenia nasion pszenicy i żołędzi u C. glareolus (Tabela 5).

Szybkość przechodzenia treści przez przewód pokarmowy badanych Microtidae nie zależy od płci. Różnice statystycznie istotne otrzymano jedynie dla wartości $R$ i czasu wydalenia $90 \%$ wskaźnika dla samców i samic M. agrestis karmionych zielonymi częściami roślin (Ryc. 3). 